\title{
Nonexistence of permutation binomials of certain shapes
}

\author{
Ariane M. Masuda* \\ Department of Mathematics and Statistics \\ University of Ottawa, Ottawa, ON K1N 6N5, Canada \\ amasuda@uottawa.ca \\ Michael E. Zieve* \\ Center for Communications Research \\ 805 Bunn Drive, Princeton, NJ 08540 \\ zieve@math.rutgers.edu
}

Submitted: Dec 23, 2006; Accepted: May 24, 2007; Published: Jun 21, 2007

Mathematics Subject Classification: $11 \mathrm{T06}$

\begin{abstract}
Suppose $x^{m}+a x^{n}$ is a permutation polynomial over $\mathbb{F}_{p}$, where $p>5$ is prime and $m>n>0$ and $a \in \mathbb{F}_{p}^{*}$. We prove that $\operatorname{gcd}(m-n, p-1) \notin\{2,4\}$. In the special case that either $(p-1) / 2$ or $(p-1) / 4$ is prime, this was conjectured in a recent paper by Masuda, Panario and Wang.
\end{abstract}

\section{Introduction}

A polynomial over a finite field is called a permutation polynomial if it permutes the elements of the field. These polynomials have been studied intensively in the past two centuries. Permutation monomials are completely understood: for $m>0, x^{m}$ permutes $\mathbb{F}_{q}$ if and only if $\operatorname{gcd}(m, q-1)=1$. However, even though dozens of papers have been written about them, permutation binomials remain mysterious. In this note we prove the following result:

Theorem 1.1. If $p>5$ is prime and $f:=x^{m}+a x^{n}$ permutes $\mathbb{F}_{p}$, where $m>n>0$ and $a \in \mathbb{F}_{p}^{*}$, then $\operatorname{gcd}(m-n, p-1) \notin\{2,4\}$.

${ }^{*}$ This work proves the conjectures stated in the first author's talk at the November 2006 BIRS workshop on Polynomials over Finite Fields and Applications. The authors thank BIRS for providing wonderful facilities. The first author was at Carleton University when this research was performed. 
In case $(p-1) / 2$ or $(p-1) / 4$ is prime, this was conjectured in the recent paper [2] by Panario, Wang and the first author. It is well-known that the ged is not 1: for in that case, $f$ has more than one root in $\mathbb{F}_{p}$, since $x^{m-n}$ is a permutation polynomial. It is much more difficult to show that the gcd is not 2 or 4 .

In Section 2 we prove some general results about permutation binomials, and in particular we show that it suffices to prove Theorem 1.1 when $m-n$ divides $p-1$. Then we prove Theorem 1.1 in Section 3.

Throughout this paper, we want to ignore permutation binomials that are really monomials in disguise. Here one can disguise a permutation monomial (over $\mathbb{F}_{q}$ ) by adding a constant plus a multiple of $x^{q}-x$; such addition does not affect the permutation property. Thus, we say a permutation binomial of $\mathbb{F}_{q}$ is trivial if it is congruent modulo $x^{q}-x$ to the sum of a constant and a monomial. In other words, the nontrivial permutation binomials are those whose terms have degrees being positive and incongruent modulo $q-1$.

\section{Permutation binomials in general}

Lemma 2.1. If $f$ is a permutation polynomial over $\mathbb{F}_{q}$, then the greatest common divisor of the degrees of the terms of $f$ is coprime to $q-1$.

Proof. Otherwise $f$ is a polynomial in $x^{d}$, where $d>1$ divides $q-1$, but $x^{d}$ is not a permutation polynomial so $f$ is not one either.

Lemma 2.2. Let $d \mid(q-1)$, and suppose there are no nontrivial permutation binomials over $\mathbb{F}_{q}$ of the form $x^{e}\left(x^{d}+a\right)$. Then there are no nontrivial permutation binomials over $\mathbb{F}_{q}$ of the form $x^{n}\left(x^{k}+a\right)$ with $\operatorname{gcd}(k, q-1)=d$.

Proof. Suppose $f(x):=x^{n}\left(x^{k}+a\right)$ permutes $\mathbb{F}_{q}$, where $n, k, a \neq 0$. Let $d=\operatorname{gcd}(k, q-1)$. Pick $r>0$ such that $k r \equiv d(\bmod q-1)$ and $\operatorname{gcd}(r, q-1)=1$. Then $f\left(x^{r}\right)$ permutes $\mathbb{F}_{q}$ and $f\left(x^{r}\right) \equiv x^{n r}\left(x^{d}+a\right)\left(\bmod x^{q}-x\right)$.

Lemma 2.2 immediately implies the following result from [2]:

Corollary 2.3. If $q-1$ is a Mersenne prime, then there are no nontrivial permutation binomials over $\mathbb{F}_{q}$.

We give one further reduction along the lines of Lemma 2.2:

Lemma 2.4. Let $d, n, e>0$ satisfy $d \mid(q-1), \operatorname{gcd}(n e, d)=1$ and $n \equiv e(\bmod (q-1) / d)$. Then $x^{n}\left(x^{d}+a\right)$ permutes $\mathbb{F}_{q}$ if and only if $x^{e}\left(x^{d}+a\right)$ does.

Proof. Write $f:=x^{n}\left(x^{d}+a\right)$ and $g:=x^{e}\left(x^{d}+a\right)$. For any $z \in \mathbb{F}_{q}$ with $z^{d}=1$, we have $f(z x)=z^{n} f(x)$; since $\operatorname{gcd}(n, d)=1$, this implies that the values of $f$ on $\mathbb{F}_{q}$ comprise all the $d^{\text {th }}$ roots of the values of $f(x)^{d}$. Since $f(x)^{d} \equiv g(x)^{d}\left(\bmod x^{q}-x\right)$, the result follows.

Finally, since we constantly use it, we give here a version of Hermite's criterion [1]: 
Lemma 2.5. A polynomial $f \in \mathbb{F}_{q}[x]$ is a permutation polynomial if and only if

1. for each $i$ with $0<i<q-1$, the reduction of $f^{i}$ modulo $x^{q}-x$ has degree less than $q-1 ;$ and

2. $f$ has precisely one root in $\mathbb{F}_{q}$.

\section{Proof of Theorem 1.1}

In this section we prove Theorem 1.1. We treat the cases of gcd 2 and 4 separately.

Theorem 3.1. If $p$ is prime and $x^{n}\left(x^{k}+a\right)$ is a nontrivial permutation binomial over $\mathbb{F}_{p}$, then $\operatorname{gcd}(k, p-1)>2$.

Proof. There are no nontrivial permutation binomials over $\mathbb{F}_{2}$ or $\mathbb{F}_{3}$, so we may assume $p=2 \ell+1$ with $\ell>1$. By Lemma 2.2, it suffices to show there are no nontrivial permutation binomials of the form $f:=x^{n}\left(x^{d}+a\right)$ with $d \in\{1,2\}$. This is clear for $d=1$ (since then $f(0)=f(-a)$ ), so we need only consider $d=2$. Assume $f:=x^{n}\left(x^{2}+a\right)$ is a permutation binomial. Lemma 2.1 implies $n$ is odd.

Suppose $\ell$ is odd. We will use Hermite's criterion with exponent $\ell-1$; to this end, we compute

$$
f^{\ell-1}=x^{n \ell-n}\left(x^{2}+a\right)^{\ell-1}=x^{n \ell-n} \sum_{i=0}^{\ell-1}\left(\begin{array}{c}
\ell-1 \\
i
\end{array}\right) a^{\ell-1-i} x^{2 i} .
$$

Write $f^{\ell-1}=\sum_{i=0}^{\ell-1} b_{i} x^{n \ell-n+2 i}$, where $b_{i}=\left(\begin{array}{c}\ell-1 \\ i\end{array}\right) a^{\ell-1-i}$. Since $\ell-1<p$ and $p$ is prime, each $b_{i}$ is nonzero. Thus, the degrees of the terms of $f^{\ell-1}$ are precisely the elements of

$$
S=\{n \ell-n, n \ell-n+2, n \ell-n+4, \ldots, n \ell-n+2 \ell-2\}
$$

Since $\ell$ is odd, $S$ consists of $\ell$ consecutive even numbers, so it contains a unique multiple of $p-1=2 \ell$. Thus the reduction of $f^{\ell-1}$ modulo $x^{p}-x$ has degree $p-1$, which contradicts Hermite's criterion.

If $\ell$ is even then $f^{\ell}=\sum_{i=0}^{\ell} c_{i} x^{n \ell+2 i}$, where each $c_{i}=\left(\begin{array}{c}\ell \\ i\end{array}\right) a^{\ell-i}$ is nonzero. The degrees of the terms of $f^{\ell}$ consist of the $\ell+1$ consecutive even numbers $n \ell, n \ell+2, \ldots, n \ell+2 \ell$. Since $n$ is odd, $n \ell$ is not a multiple of $p-1=2 \ell$. Thus $f^{\ell}$ has a unique term of degree divisible by $p-1$, which again contradicts Hermite's criterion.

Theorem 3.2. If $p$ is prime and $x^{n}\left(x^{k}+a\right)$ is a nontrivial permutation binomial over $\mathbb{F}_{p}$, then $\operatorname{gcd}(k, p-1) \neq 4$.

Proof. Plainly we need only consider primes $p$ with $p \equiv 1(\bmod 4)$. By Lemma 2.2, it suffices to show there are no nontrivial permutation binomials of the form $x^{n}\left(x^{4}+a\right)$. By Lemma 2.1, we may assume $n$ is odd. By Lemma 2.4, it suffices to show nonexistence with $0<n<(p-1) / 4$ if $p \equiv 1(\bmod 8)$, and with $0<n<(p-1) / 2$ if $p \equiv 5(\bmod 8)$. Assume $f:=x^{n}\left(x^{4}+a\right)$ is a nontrivial permutation binomial with $n$ satisfying these constraints. 
First suppose $p \equiv 1(\bmod 8)$, say $p=8 \ell+1$; here our assumption is $0<n<2 \ell$. The set of degrees of terms of $f^{2 \ell}$ is

$$
S=\{2 \ell n, 2 \ell n+4,2 \ell n+8, \ldots, 2 \ell n+8 \ell\} .
$$

When $\ell$ is even, $S$ consists of $2 \ell+1$ consecutive multiples of 4 . Since $n$ is odd, $2 \ell n$ is not a multiple of $8 \ell$, so $S$ contains precisely one multiple of $p-1=8 \ell$, contradicting Hermite's criterion. So assume $\ell$ is odd; since $8 \ell+1$ is prime, we have $\ell \geq 5$. Now the set of degrees of terms of $f^{2 \ell+2}$ is

$$
S=\{2 \ell n+2 n, 2 \ell n+2 n+4,2 \ell n+2 n+8, \ldots, 2 \ell n+2 n+4(2 \ell+2)\} .
$$

Here $S$ consists of $2 \ell+3$ consecutive multiples of 4 , so it contains a multiple of $p-1=8 \ell$. By Hermite's criterion, $S$ must have at least two such multiples. Thus, $8 \ell$ divides either $2 \ell n+2 n, 2 \ell n+2 n+4$ or $2 \ell n+2 n+8$, so $\ell$ divides either $n, n+2$ or $n+4$. Since $\ell \geq 5$ and $0<n<2 \ell$, we have $n+4<3 \ell$; since $n$ is odd, it follows that $\ell$ equals either $n, n+2$ or $n+4$. But then $f^{8}$ has a unique term of degree divisible by $p-1=8 \ell$, contradicting Hermite's criterion.

Thus we have $p \equiv 5(\bmod 8) ;$ write $p=4 \ell+1$ with $\ell$ odd, where again $0<n<2 \ell$. Suppose $\ell \equiv 1(\bmod 4)$. If $\ell=1$ then $f$ is trivial, so assume $\ell>1$. The set of degrees of terms of $f^{\ell-1}$ is

$$
S=\{n \ell-n, n \ell-n+4, n \ell-n+8, \ldots, n \ell-n+4 \ell-4\} .
$$

Since $\ell \equiv 1(\bmod 4)$, the set $S$ consists of $\ell$ consecutive multiples of 4 , so $S$ contains precisely one multiple of $p-1=4 \ell$, contradicting Hermite's criterion.

Thus $\ell \equiv 3(\bmod 4)$. The set of degrees of terms of $f^{\ell+1}$ is

$$
S=\{n \ell+n, n \ell+n+4, n \ell+n+8, \ldots, n \ell+n+4 \ell+4\} .
$$

Since $S$ consists of $\ell+2$ consecutive multiples of 4 , it certainly contains a multiple of $4 \ell$, so (by Hermite's criterion) it must contain two such multiples. Thus either $n(\ell+1)$ or $n(\ell+1)+4$ is a multiple of $4 \ell$, so $\ell$ divides either $n$ or $n+4$. Since $n$ is odd and $0<n<2 \ell$, the only possibilities are $n=\ell$ or $n=\ell-4$ or $(n, \ell)=(5,3)$. If $n=\ell-4$ then $f^{4}$ has degree $4 \ell=p-1$, contradicting Hermite's criterion. If $(n, \ell)=(5,3)$, then $p=13$ and $a^{-1} f\left(x^{11}\right)$ permutes $\mathbb{F}_{p}$; since $a^{-1} f\left(x^{11}\right) \equiv x^{3}\left(x^{4}+a^{-1}\right)\left(\bmod x^{13}-x\right)$, it suffices to treat the case $n=\ell$. Finally, suppose $n=\ell$, so $f=x^{\ell}\left(x^{4}+a\right)$ permutes $\mathbb{F}_{p}$. The degrees of the terms of $f^{4}$ are

$$
4 \ell, 4 \ell+4,4 \ell+8,4 \ell+12,4 \ell+16 .
$$

We have our usual contradiction if the degree $4 \ell$ term is the unique term of $f^{4}$ with degree divisible by $4 \ell$, so the only remaining possibility is that $4 \ell$ divides either $4,8,12$ or 16 . Since $\ell \equiv 3(\bmod 4)$, the only possibility is $\ell=3$. Finally, when $\ell=3$, the coefficient of $x^{12}$ in the reduction of $f^{4}$ modulo $x^{13}-x$ is $a^{4}+4 a$, which must be zero (by Hermite), so $a^{3}=-4$; but the cubes in $\mathbb{F}_{13}^{*}$ are \pm 1 and \pm 8 , contradiction. 


\section{References}

[1] Ch. Hermite, Sur les fonctions de sept lettres, C. R. Acad. Sci. Paris 57 (1863), 750757.

[2] A. Masuda, D. Panario, and Q. Wang, The number of permutation binomials over $\mathbb{F}_{4 p+1}$ where $p$ and $4 p+1$ are primes, Electronic J. Combin. 13 (2006), R65. 